\title{
Hard-sphere fluids inside spherical, hard pores. Grand canonical ensemble Monte Carlo calculations and integral equation approximations
}

Sloth, Peter

Published in:

Journal of Chemical Physics

Link to article, DOI:

10.1063/1.459141

Publication date:

1990

Document Version

Publisher's PDF, also known as Version of record

Link back to DTU Orbit

Citation (APA):

Sloth, P. (1990). Hard-sphere fluids inside spherical, hard pores. Grand canonical ensemble Monte Carlo calculations and integral equation approximations. Journal of Chemical Physics, 93(2), 1292-1298.

https://doi.org/10.1063/1.459141

\section{General rights}

Copyright and moral rights for the publications made accessible in the public portal are retained by the authors and/or other copyright owners and it is a condition of accessing publications that users recognise and abide by the legal requirements associated with these rights.

- Users may download and print one copy of any publication from the public portal for the purpose of private study or research.

- You may not further distribute the material or use it for any profit-making activity or commercial gain

- You may freely distribute the URL identifying the publication in the public portal 


\title{
Hard-sphere fluids inside spherical, hard pores. Grand canonical ensemble Monte Carlo calculations and integral equation approximations
}

\author{
Peter Sloth \\ Fysisk-Kemisk Institut, Technical University of Denmark, Bldg. 206, DK 2800 Lyngby, Denmark
}

(Received 8 February 1990; accepted 26 March 1990)

\begin{abstract}
Density profiles and partition coefficients are obtained for hard-sphere fluids inside hard, spherical pores of different sizes by grand canonical ensemble Monte Carlo calculations. The Monte Carlo results are compared to the results obtained by application of different kinds of integral equation approximations. Also, some exact, analytical results for the partition coefficients are given, which are valid in the case of (very) small pores or at low density, respectively.
\end{abstract}

\section{INTRODUCTION}

Recently, Zhou and Stell discussed the application of different kinds of integral equation approximations for fluids inside pores of different shapes. ${ }^{1,2}$ The subject of the present work is to investigate how well these approximations work in the case of a simple fluid inside spherical pores. For this purpose, a number of grand canonical ensemble Monte Carlo (GCEMC) calculations ${ }^{3}$ were carried out for hardsphere fluids inside hard spherical pores. The GCEMC approach was chosen, because the results from this method may be directly compared with the integral equation approaches of Ref. 1.

This paper is organized as follows: In Sec. II, the model is introduced, and some exact relations/results are given. In Sec. III, we discuss the different integral equation approximations and their application to hard sphere fluids inside spherical pores. In Sec. IV, a description of the GCEMC method is given. Finally, a comparison between integral equation approximation results and $\mathrm{MC}$ data is given in Sec. $\mathrm{V}$, and the main findings are summarized in the conclusion (Sec. VI).

\section{THE MODEL AND SOME EXACT RESULTS}

Following Zhou and Stell, ${ }^{1}$ we consider a system of a (one component) hard-sphere fluid inside a hard spherical pore as a special case of a two-component system. One of the species-which is denoted $m$-is made up of ordinary hard spheres, with diameter $d$, which model the fluid. The interaction between two particles of species $m$ is thus given by the well known hard-sphere pair potential function

$$
u_{m m}(r)=\left\{\begin{array}{cc}
\infty, & r<d \\
0, & r \geqslant d
\end{array}\right.
$$

The other species-which represent hard pores of radius $R$ and is denoted by $p$-is made up of spherical hard shells with an inner surface of radius $R$ and with an outer surface of radius $R+l$. The interaction between a particle of species $m$ and a particle of species $p$ is given by

$$
u_{p m}(r)= \begin{cases}0, & r \leqslant R-d / 2 \\ \infty, & R-d / 2<r<R+l+d / 2 . \\ 0, & R+l+d / 2 \leqslant r\end{cases}
$$

The inner cavity of a particle of species $p$ models a spherical hard pore. In the pore model given by application of Eq. (2.2) within the framework of statistical mechanics, the hard-sphere particles, of which the fluid is composed, may in principle "jump" in and out of the pore. That is, the particles inside the pores are in equilibrium with the particles outside the pores. However, a particle is never in the act of passing a pore wall, which should be possible in a more realistic model. (The same picture is not quite true in, for example, molecular dynamic simulations, because the number of particles inside the pores in this case always would be fixed by the starting configuration.) In the calculations, we consider a single pore. That is, we let the particle density $\rho_{p}$ of the species $p$ approach zero. In this limit, the behavior of the species $m$ becomes identical to that of a homogeneous system-in equilibrium with the fluid inside the pore-as the distance from the "pore particle" becomes large. Also, the outer shape of the pore particle, e.g., the thickness of the pore wall $(l)$ should not influence the final results obtained for the properties inside the pore. Furthermore, as the volume of the total system (pore particle and hard-sphere particles) goes to infinity, the average particle density of the species $m$, $\rho_{m}$, will be that of the homogeneous system in equilibrium with the fluid inside the pore.

Many modern statistical mechanical approximations are based on the Ornstein-Zernike $(\mathrm{OZ})$ equation, ${ }^{4}$ which defines the direct correlation functions $c_{i j}(\mathbf{r})$. In the limit $\rho_{p} \rightarrow 0$, the $\mathrm{OZ}$ equation (s) can be written as

$h_{m m}\left(\mathbf{r}_{12}\right)-c_{m m}\left(\mathbf{r}_{12}\right)=\rho_{m} \int c_{m m}\left(\mathbf{r}_{13}\right) h_{m m}\left(\mathbf{r}_{32}\right) d \mathbf{r}_{3}$,

$h_{p m}\left(\mathbf{r}_{12}\right)-c_{p m}\left(\mathbf{r}_{12}\right)=\rho_{m} \int c_{m m}\left(\mathbf{r}_{13}\right) h_{p m}\left(\mathbf{r}_{32}\right) d \mathbf{r}_{3}$,

or,

$g_{p m}\left(\mathbf{r}_{12}\right)=c_{p m}\left(\mathbf{r}_{12}\right)+a_{1}+\rho_{m} \int c_{m m}\left(\mathbf{r}_{13}\right) g_{p m}\left(\mathbf{r}_{32}\right) d \mathbf{r}_{3}$,

with

$$
a_{1} \equiv 1-\rho_{m} \int c_{m m}(\mathbf{r}) d \mathbf{r},
$$

where $h_{i j}(r) \equiv g_{i j}(r)-1$ is the total correlation function between a particle of species $i$ and a particle of species $j$. The 
product $\rho_{m} g_{p m}(r)$ measures the local concentration of the species $m$ inside the pore, with $r=0$ corresponding to the center of the pore.

In passing, it is noted that Eq. (2.2) implies that $g_{p m}(r)=0$ if $R-d / 2<r<R+l+d / 2$.

Equations (2.3)-(2.5) are formally exact, and can be used in connection with different kinds of closure approximations for the direct and total correlation functions. In Sec. III, we discuss two integral equation approximations, which are based on Eqs. (2.3)-(2.5).

The average number of particles $\langle N\rangle_{p}$ inside the pore is calculated from

$$
\langle N\rangle_{\rho}=4 \pi \rho_{m} \int_{0}^{R-d / 2} g_{p m}(r) r^{2} d r .
$$

Similarly, the average density $\left\langle\rho_{m}\right\rangle_{p}$ of particles inside the pore can be given by

$$
\left\langle\rho_{m}\right\rangle_{p}=3\langle N\rangle_{p} / 4 \pi R^{3}=K \rho_{m}
$$

with

$$
\begin{aligned}
K & =\frac{3\langle N\rangle_{p}}{4 \pi R^{3} \rho_{m}} \\
& =K_{0}+\left(3 / R^{3}\right) \int_{0}^{R-d / 2}\left[g_{p m}(r)-1\right] r^{2} d r \\
K_{0} & =(1-d / 2 R)^{3}
\end{aligned}
$$

where $K$ is a partition coefficient, and $K_{0}$ is equivalent to a Henry-law constant if $R>d / 2$.

If $R<d$, the problem can be solved exactly and the result for $g_{p m}(r)$ is derived in the Appendix. Using Eq. (A7) from the Appendix and Eq. (2.8), we find the following exact results for $K$ :

$$
\begin{aligned}
& K=0 \quad \text { if } \quad R<d / 2, \\
& K=\left(z / \rho_{m}\right) \frac{K_{0}}{1+(4 \pi / 3) \tau z d^{3}} \text { if } d / 2<R<d,
\end{aligned}
$$

where $z$ is the activity of the hard-sphere fluid, and with the definition

$$
\tau \equiv R / d-0.5 \text {. }
$$

In the limit of low concentrations, we have the expression for $g_{p m}(r):^{5}$

$$
\begin{aligned}
g_{p m}(\mathbf{r})= & \exp \left[-\beta u_{p m}(\mathbf{r})\right]\left[1+y_{1}^{(1)}(\mathbf{r})\right. \\
& \left.\times \rho_{m}+o\left(\rho_{m}\right)\right]
\end{aligned}
$$

with

$$
y_{1}^{(1)}\left(\mathbf{r}_{12}\right)=\int f_{p m}\left(\mathbf{r}_{13}\right) f_{m m}\left(\mathbf{r}_{32}\right) d \mathbf{r}_{3} .
$$

Here, $\beta \equiv 1 / k_{B} T$ ( $k_{B}$ being the Boltzmann constant and $T$ the absolute temperature), and $f_{i j}(\mathbf{r})$ is the Mayer $f$ function, defined by

$$
f_{i j}(\mathbf{r}) \equiv \exp \left[-\beta u_{i j}(\mathbf{r})\right]-1 .
$$

Using the pair-potential functions given by Eqs. (2.1) and (2.2), the following expressions for $y_{1}^{(1)}(r)$ are obtained for $r \leqslant R-0.5 d$ :

$y_{1}^{(1)}(r)=A$ if $R \leqslant d$,

$y_{1}^{(1)}(r)=\left\{\begin{array}{ll}A ; & r \leqslant 1.5 d-R \\ \psi(r) ; & r>1.5 d-R\end{array}\right.$ if $d<R \leqslant 1.5 d$,

$$
y_{1}^{(1)}(r)=\left\{\begin{array}{ll}
0 ; \quad r \leqslant R-1.5 d \\
\psi(r) ; \quad r>R-1.5 d
\end{array} \text { if } R>1.5 d\right.
$$

with

$$
\begin{aligned}
A \equiv & (4 \pi / 3) d^{3}\left(1-\tau^{3}\right), \\
\psi(r) \equiv & \frac{\pi d^{3}}{(r / d)}\left[\frac{1}{4}\left(1-\tau^{2}\right)^{2}+\frac{2}{3}(r / d)\left(1-\tau^{3}\right)\right. \\
& \left.+\frac{1}{2}(r / d)^{2}\left(1+\tau^{2}\right)-\frac{1}{12}(r / d)^{4}\right] .
\end{aligned}
$$

From Eqs. (2.8), (2.13), and (2.16), one may obtain expressions for the partition coefficients in the limit of low $\rho_{m}:$

$$
\begin{aligned}
K= & 0 \quad \text { if } 0<R \leqslant 0.5 d, \\
K= & K_{0}\left[1+(4 \pi / 3) \rho_{m} d^{3}\left(1-\tau^{3}\right)+o\left(\rho_{m}\right)\right] \\
& \text { if } 0.5 d<R \leqslant d, \\
K= & K_{0}\left[1+(3 \pi / 4) \rho_{m} d^{3} \frac{\tau^{2}-\frac{1}{18}}{\tau^{3}}+o\left(\rho_{m}\right)\right] \\
& \text { if } R>d .
\end{aligned}
$$

\section{INTEGRAL EQUATION APPROXIMATIONS}

The (exact) equations (2.3)-(2.5) are used as a starting point for two of the approximations proposed in Ref. 1. First, it is noted that Eq. (2.3) is equivalent to the $\mathrm{OZ}$ equation for the homogeneous one-component hard-sphere fluid which is in equilibrium with the fluid inside the pore. A convenient, analytical expression for $c_{m m}(r)$-used in Ref. $1-$ is provided by the Percus-Yevick (PY) approximation. ${ }^{6.7}$ The PY approximation is known to yield very reasonable results for homogeneous hard-sphere systems.

If the PY approximation is used for $c_{m m}(r)$, Eq. (2.4) might for the present system be given by $(0<r \leqslant R-d / 2)$

$g_{p m}(r)=c_{p m}(r)+a_{1}+\frac{2 \pi \rho_{m}}{r} \int_{0}^{R-d / 2} K(r, t) g_{p m}(t) t d t$,

$K(r, t)=\int_{|r-t|}^{r+t} c_{m m}^{\mathrm{PY}}(s) s d s$

with

$$
a_{1}=(1+2 \eta)^{2} /(1-\eta)^{4}
$$

$$
c_{m m}^{\mathrm{PY}}(r)=\left\{\begin{array}{c}
\frac{-(1+2 \eta)^{2}+6 \eta(1+\eta / 2)^{2}(r / d)-(1 / 2) \eta(1+2 \eta)^{2}(r / d)^{3}}{(1-\eta)^{4}} ; \quad r<d \\
0 ; \quad r \geqslant d
\end{array}\right.
$$


where $\eta \equiv(\pi / 6) \rho_{m} d^{3}$ is the packing fraction.

Given a relation between $c_{p m}(r)$ and $g_{p m}(r)$, Eq. (3.1) may be solved, by iteration, to yield $g_{p m}(r)$ inside the pore. Two such relations were considered by Zhou and Stell, ${ }^{1}$ namely the $\mathrm{PY}$ and $\mathrm{HNC}^{8}$ approximations. These approximations are, for the present systems, given by

$$
\begin{aligned}
c_{p m}^{\mathrm{PY}}(r)=0 ; \quad & \quad r \leqslant R-d / 2, \\
c_{p m}^{\mathrm{HNC}}(r)= & g_{p m}(r)-1-\ln \left[g_{p m}(r)\right] ; \\
& r \leqslant R-d / 2 .
\end{aligned}
$$

The approximations obtained by using Eq. (3.5) or Eq. (3.6) for $c_{p m}(r)$ in Eq. (3.1) are the PY/PY and the HNC/ $\mathbf{P Y}$ approximation, respectively.

Another approximation, considered in Ref. 1, is the socalled BBGKY-SA which is based on a kind of superposition approximation closure for the first member of the BBGKY hierarchy. The BBGKY-SA is given by the following equations:

with

$$
\begin{aligned}
L(r, t) & \equiv \int_{|r-t|}^{r+t} G(s) s d s \\
& = \begin{cases}0 & \text { if }|r-t| \geqslant d \\
-\left[g_{m m}(d) / 2\right]\left\{(\min [d, r+t])^{2}-(r-t)^{2}\right\} \quad \text { if }|r-t|<d\end{cases}
\end{aligned}
$$

An accurate expression for $g_{m m}(d)$ might be obtained from the Carnahan-Starling equation of state, ${ }^{9}$ i.e.,

$$
g_{m m}(d) \simeq \frac{1-\eta / 2}{(1-\eta)^{3}}
$$

\section{GCEMC CALCULATIONS}

We consider a one-component system with fixed volume $(V)$, temperature $(T)$, and chemical potential $(\mu)$. The system may be considered to consist of $B$ uniformly distributed discrete sites. (This is consistent with computer calculations, because the particle coordinates are represented by a finite number of digits.) Let $\mathbf{x}_{i}$ denote a given configuration $i$ of the system; let $N_{i}$ be the number of particles in this configuration and let $U\left(\mathbf{x}_{i}\right)$ be the corresponding total potential energy. The probability $u_{i}$ of configuration $x_{i}$ in the grand canonical ensemble is then given by

$$
u_{i}=\left(\Delta V / \lambda^{3}\right)^{N_{i}} \frac{\exp \left\{\beta\left[N_{i} \mu-U\left(\mathbf{x}_{i}\right)\right]\right\}}{\Xi(V, T, \mu)}
$$

with

$$
\Delta V=V / B
$$

In Eq. (4.1), $\Xi(V, T, \mu)$ is the grand canonical partition function and $\lambda=h /\left(2 \pi m k_{B} T\right)^{1 / 2}$ ( $h$ and $m$ being the Planck's constant and the mass of a particle, respectively). Also, it is assumed that the particles are unlabeled.

Equation (4.1) is to be used in a Markov chain. ${ }^{10}$ The steps in the chain are generated such that a given configuration $\mathbf{x}_{i}$ occurs with a probability proportional to $u_{i}$ as the length of the chain goes to infinity. The Markov chain is

$$
\begin{aligned}
\ln \left[g_{p m}\left(\mathbf{r}_{12}\right)\right]= & -\beta u_{p m}\left(\mathbf{r}_{12}\right)+C \\
& +\rho_{m} \int G\left(\mathbf{r}_{13}\right) g_{p m}\left(\mathbf{r}_{32}\right) d \mathbf{r}_{3}
\end{aligned}
$$

with

$$
\begin{aligned}
& G(r)=\left\{\begin{array}{cc}
-g_{m m}(d) ; \quad r \leqslant d \\
0 ; \quad r>d
\end{array},\right. \\
& C \equiv-\rho_{m} \int G(r) d \mathbf{r}=8 \eta g_{m m}(d) .
\end{aligned}
$$

[Note that the definition of the function $G(r)$ given here differs slightly from that used in Ref. 1.]

For numerical calculations, it is convenient to rewrite Eq. (3.7) as $(0<r \leqslant R-d / 2)$

$$
\ln \left[g_{p m}(r)\right]=C+\frac{2 \pi \rho_{m}}{r} \int_{0}^{R-d / 2} L(r, t) g_{p m}(t) t d t
$$

specified by one-step probabilities $p_{i j}$ of transition from configuration $\mathbf{x}_{i}$ to configuration $x_{j}$. Here, we require that these transition probabilities satisfies microscopic reversibility, i.e.,

$$
u_{i} p_{i j}=u_{j} p_{j i}
$$

with the normalizing condition

$$
\Sigma_{j} p_{i j}=1 \text {. }
$$

Let $q_{i j}$ be the probability of attempting a transition from configuration $\mathbf{x}_{i}$ to configuration $\mathbf{x}_{j}(i \neq j)$, and let $f_{i j}$ be the probability that this attempted move is accepted. Then $p_{i j}$ $=q_{i j} f_{i j}$, and Eq. (4.3) is equivalent to

$$
f_{i j} / f_{j i}=u_{j} q_{j i} / u_{i} q_{i j}
$$

In our program, the generation of a new configuration from a former was made by the following procedure: Addition of a particle at a randomly chosen position or deletion of a randomly chosen particle was attempted with equal probability. Let us suppose that configuration $\mathbf{x}_{i}$ is composed by $N_{i}$ particles, and that configuration $\mathbf{x}_{j}$ is composed by $N_{j}=N_{i}+1$ particles (the positions of the $N_{i}$ particles being the same in both of the configurations). In this case, $q_{i j}$ and $q_{j i}$ are given by

$$
\begin{aligned}
& q_{i j}=1 / 2 B, \\
& q_{j i}=1 / 2 N_{j}=1 / 2\left(N_{i}+1\right) .
\end{aligned}
$$

Combination of Eqs. (4.1) and (4.5)-(4.7) gives 


$$
\begin{aligned}
\frac{f_{i j}}{f_{j i}} & =\frac{V}{N_{j}} z \exp \left\{-\beta\left[U\left(\mathbf{x}_{j}\right)-U\left(\mathbf{x}_{i}\right)\right]\right\} \\
& \equiv F(i, j)
\end{aligned}
$$

with

$$
z \equiv \exp (\beta \mu) / \lambda^{3}
$$

where $z$ is the activity which, in our case, is determined by a homogeneous hard-sphere system with density $\rho_{m}$ and hardsphere diameter $d$. That is

$$
z=\rho_{m} \exp \left(\beta \mu^{\mathrm{ex}}\right) \text {. }
$$

In Eq. (4.10), $\mu^{\text {ex }}$ is the excess chemical potential of the homogeneous hard-sphere fluid. In our calculations, we have used the Carnahan-Starling equation of state for the calculation of this property ${ }^{9}$ :

$$
\beta \mu^{\mathrm{ex}} \simeq \eta\left(8-9 \eta+3 \eta^{2}\right) /(1-\eta)^{3} .
$$
ting

Equation (4.8) was used in our MC calculations by set$f_{i j}=\left\{\begin{array}{cc}1 & \text { if } F(i, j) \geqslant 1 \\ F(i, j) & \text { if } F(i, j)<1\end{array}\right.$ for addition $f_{j i}=\left\{\begin{array}{c}1 \text { if } 1 / F(i, j)>1 \\ 1 / F(i, j) \quad \text { if } 1 / F(i, j) \leqslant 1\end{array}\right.$ for deletion

which is the procedure commonly applied.

\section{NUMERICAL RESULTS}

Two series of MC runs were carried out. In the first series, $\rho_{m}^{*} \equiv \rho_{m} d^{3}$ was kept fixed at $\rho_{m}^{*}=0.5$ and $R / d$ was varied between $R / d=1.25$ and $R / d=5.0$. In the second series of runs, $R / d$ was held fixed at $R / d=2$ and $\rho_{m}^{*}$ was varied between $\rho_{m}^{*}=0.1$ and $\rho_{m}^{*}=0.6$. The parameters used in the MC calculations are summarized in Table $I$ together with the results for $\langle N\rangle_{p}$ and $K / K_{0}$. The partition coefficient from Table $I$ are plotted in Figs. 1 and 2 together with results obtained from the PY/PY, HNC/PY, and BBGKY-SA approximations. For comparison are also given the corresponding curves as given by the low-density limit, cf. Eq. (2.17), that is

TABLE I. Grand canonical Monte Carlo results for $\langle N\rangle_{\rho}$ and $K / K_{0}$ for different values of $R / d$ and $\rho_{m}^{*}$.

\begin{tabular}{lcccc}
\hline \hline$R / d$ & $\rho_{m}^{*}$ & $\begin{array}{c}\text { Total }^{\mathrm{a}} \text { no. of } \\
{\text { config. } \times 10^{-6}}^{*}\end{array}$ & $\langle N\rangle_{p}$ & $K / K_{0}$ \\
\hline 1.25 & 0.5 & 30 & 2.220 & 2.513 \\
1.5 & 0.5 & 15 & 4.387 & 2.095 \\
2.0 & 0.1 & 30 & 1.629 & 1.152 \\
2.0 & 0.2 & 30 & 3.664 & 1.296 \\
2.0 & 0.3 & 30 & 6.058 & 1.428 \\
2.0 & 0.4 & 30 & 8.772 & 1.551 \\
2.0 & 0.5 & 30 & 11.77 & 1.665 \\
2.0 & 0.6 & 120 & 14.84 & 1.750 \\
2.5 & 0.5 & 30 & 24.62 & 1.469 \\
3.5 & 0.5 & 70 & 73.36 & 1.297 \\
5.0 & 0.5 & 100 & 227.4 & 1.191 \\
\hline
\end{tabular}

The first 10000 configurations were neglected in the calculations of $\langle N\rangle_{p}$.

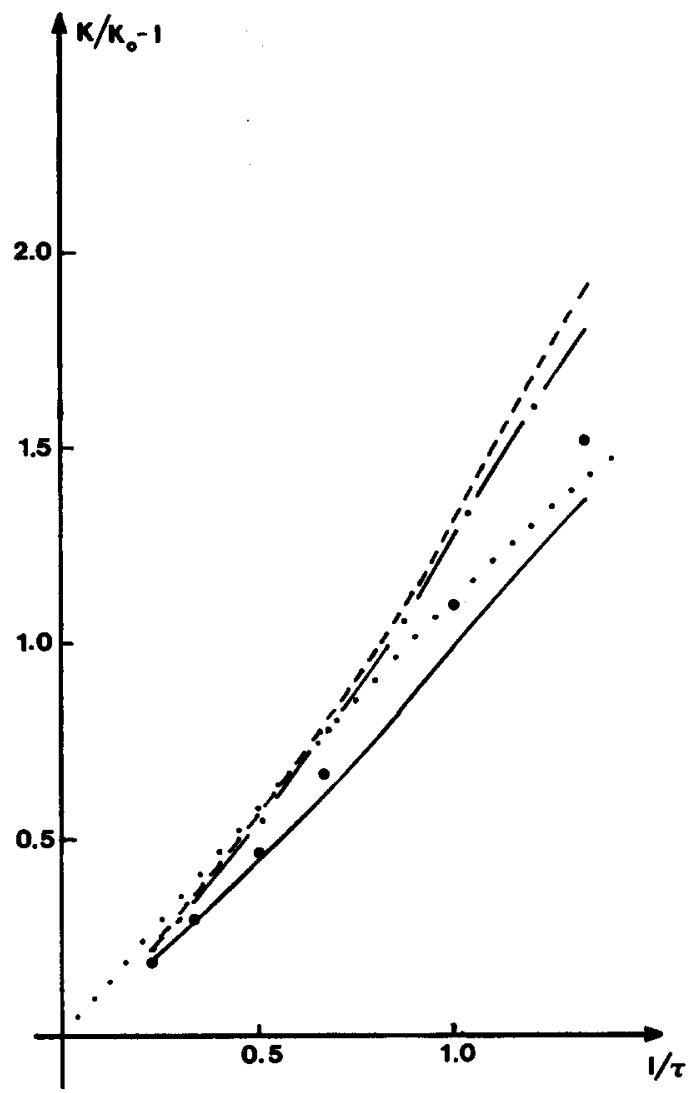

FIG. $1 . K / K_{0}$ as a function of $\tau^{-1}$ for $p_{m}^{*}=0.5$. Filled circles are the GCEMC results; (-): PY/PY; (--): HNC/PY; (--): BBGKY-SA; $(\cdots)$ : Eq. (5.1).

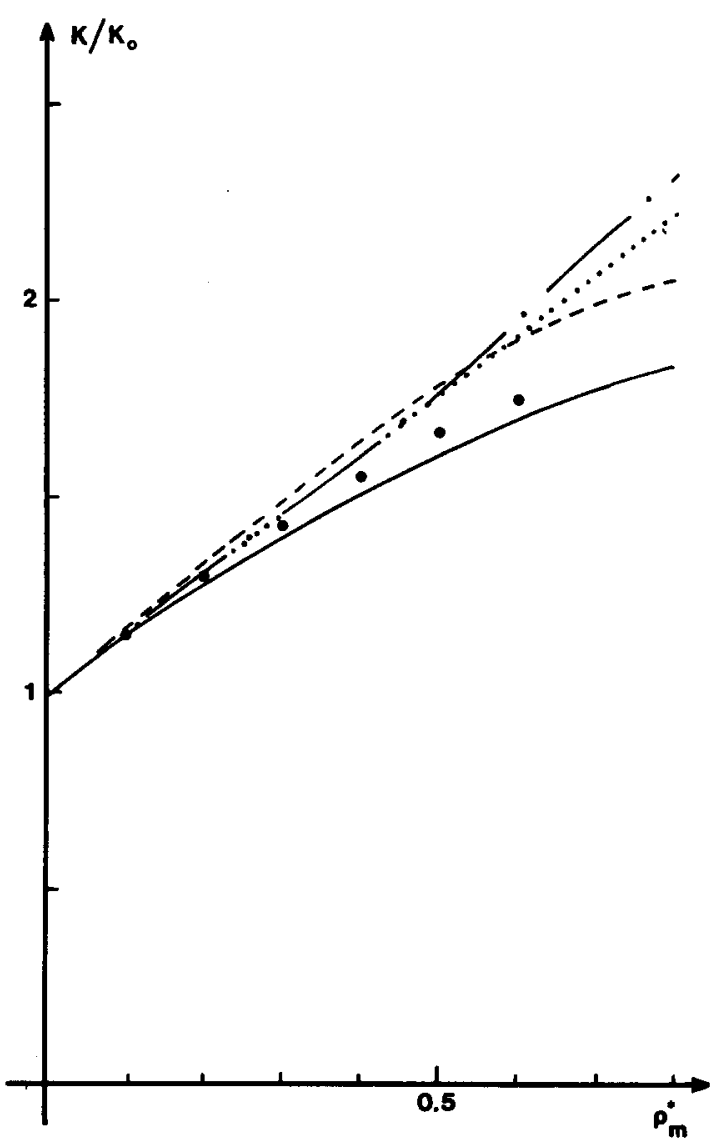

FIG. 2. $K / K_{0}$ as a function of $\rho_{m}^{*}$ for $R / d=2.0$. The symbols are as in Fig 1. 


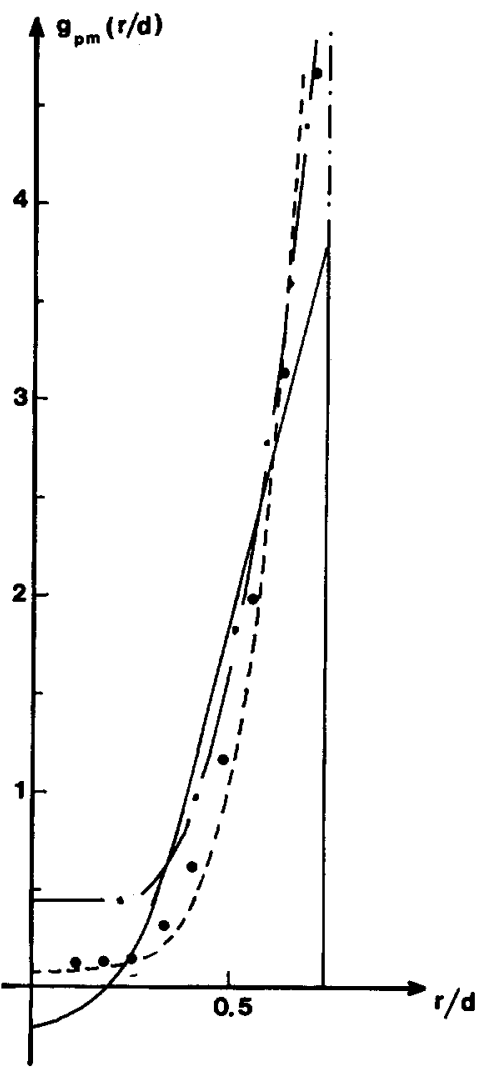

(a)
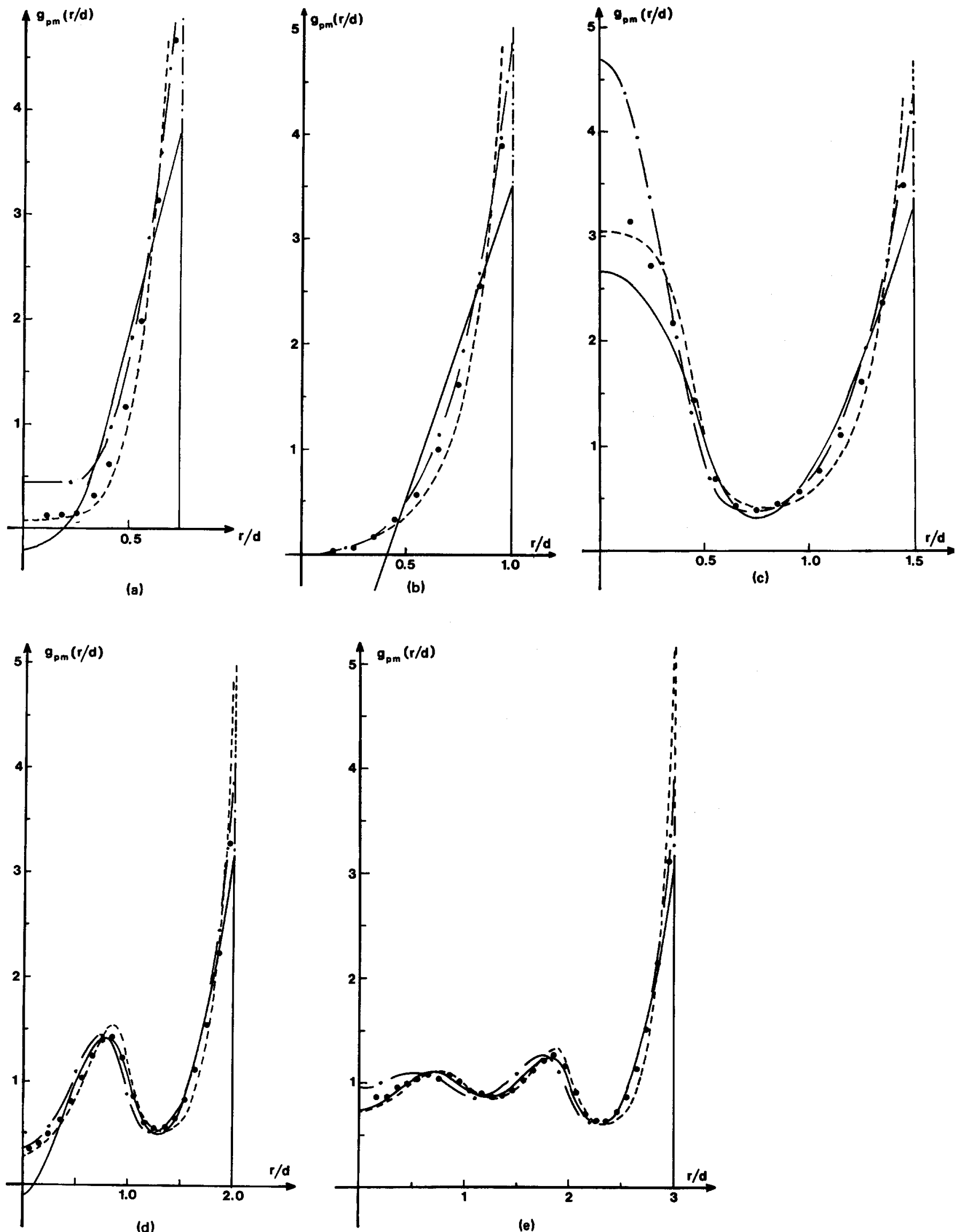

(d)

(e)

FIG. 3. The density profile $g_{p m}(r)$, inside a number of spherical pores with $\rho_{m}^{*}=0.5$. Filled circles are the GCEMC results; ( - ): PY/PY; (--): HNC/PY; (-·): BBGKY-SA. (a) $R / d=1.25$; (b) $R / d=1.5$; (c) $R / d=2.0$; (d) $R / d=2.5$; (e) $R / d=3.5$; (f) $R / d=5.0$. 


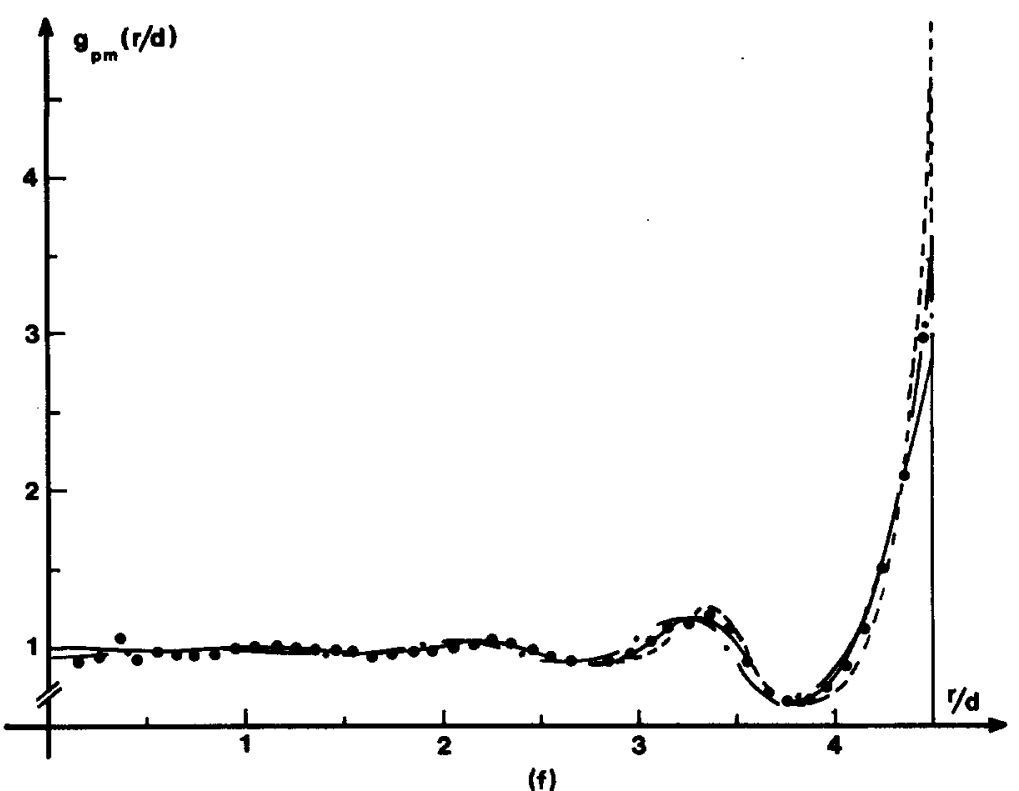

FIG. 3 (continued).

$$
K / K_{0}=1+(3 \pi / 4) \rho_{m}^{*} \frac{\tau^{2}-1 / 18}{\tau^{3}} ; \quad R>d .
$$

It is seen that the PY/PY approximation-in these cases- reproduces the MC data better than does the HNC/ PY and the BBGKY-SA. Moreover, the low-density approximation, as given by Eq. (5.1), yields partition coefficients which are of comparative precision with the HNC/ PY and BBGKY-SA, over a wide range of parameters-

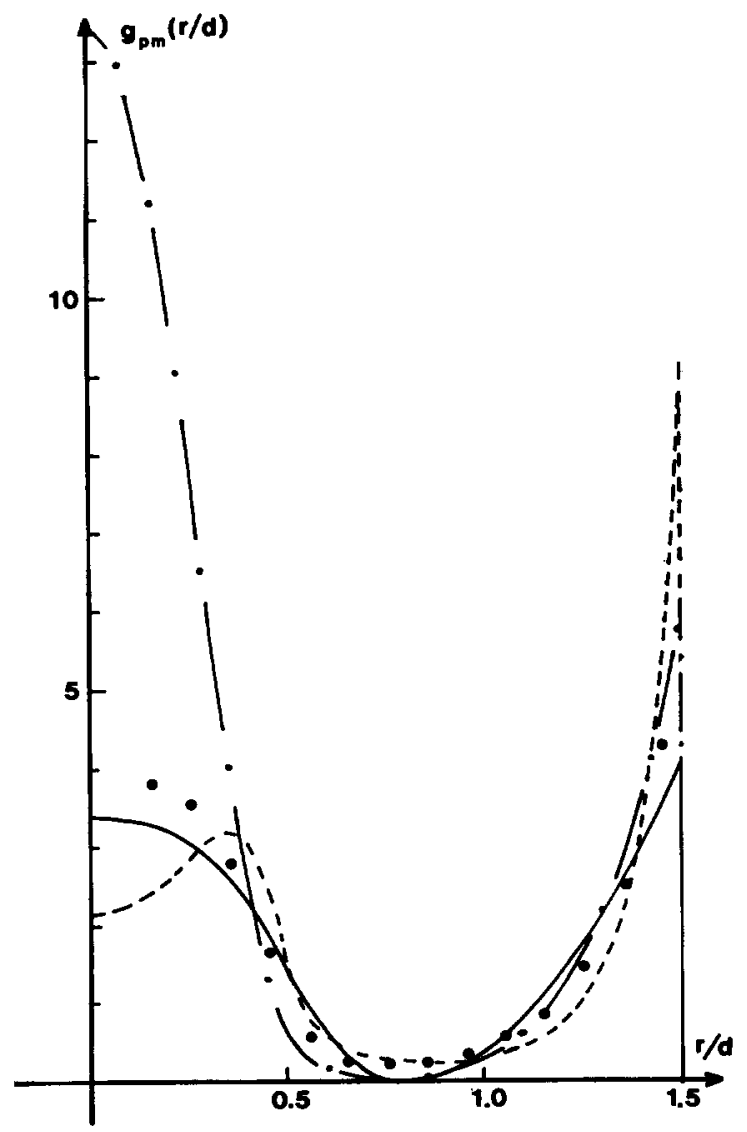

FIG. 4. The density profile $g_{p m}(r)$, inside a spherical pore with $R / d=2.0$ and $\rho_{m}^{*}=0.6$. The symbols are as in Fig. 3 . even at relatively high densities.

Results for $g_{p m}(r)$ are shown in Figs. 3(a)-3(f) for $\rho_{m}^{*}=0.5$ and the various values of $R / d$. It is seen that the PY/PY approximation-for some values of $R / d$-yields negative $g_{p m}(r)$ results at the center of the pore which, of course, have no physical meaning. In general, it is found that the values of $g_{p m}(r)$, near the center of the pore, are underestimated by the PY/PY approximation (except for $R$ / $d=5.0$ ) and, for a number of cases, overestimated by the BBGKY-SA. Also, the PY/PY approximation is found to yield results for $g_{p m}(r)$ which are too low near the pore wall, whereas the corresponding HNC/PY results are too high in this case. All in all, however, all three approximations reproduces the correct shapes of the curves reasonably well for all cases shown in Fig. 3. The same picture is not quite true, however, if we, for example, increase the value of $\rho_{m}^{*}$. In Fig. 4 , we show the density profiles obtained for $R / d=2.0$ and the slightly higher density $\rho_{m}^{*}=0.6$. It is seen that the PY/ PY and BBGKY-SA curves show a maximum at $r=0$, in agreement with the $\mathrm{MC}$ results [although the values of $g_{p m}(r)$ at $r \approx 0$ are greatly overestimated by the BBGKYSA]. In the case of the HNC/PY approximation, however, this maximum is displaced to $r \simeq 0.35 d$.

\section{CONCLUSION}

We conclude that the PY/PY approximation seems to be the best among the integral equation approximations, considered in this work, to predict the partition coefficients. However, none of the approximations can be said to give the best overall description of the density profiles inside the pores.

\section{APPENDIX}

The grand canonical partition function $\Xi(V, T, \mu)$ may, in general, be given as ${ }^{11}$

$$
\Xi(V, T, \mu)=1+\sum_{N=1}^{\infty} Q_{N}(V, T) \exp (N \beta \mu),
$$

where $Q_{N}(V, T)$ is the canonical partition function for a $N$ - 
particle system. If the pore radius is smaller than $R=d$, the largest possible number of particles inside the pore is one. Furthermore, if $R<d / 2$, no particles can be present in the pore and we have the trivial result $g_{p m}(r)=0$ inside the pore. If $R$ is in the interval $d / 2<R<d$, Eq. (A1) yields

$$
\begin{aligned}
\Xi(V, T, \mu) & =1+Q_{1}(V, T) \exp (\beta \mu) \\
& =1+\left(e^{\beta \mu} / \lambda^{3}\right) \int \exp \left[-\beta u_{1}(\mathbf{r})\right] d \mathbf{r},(\mathrm{A} 2)
\end{aligned}
$$

where $u_{1}(\mathbf{r})$ is the one-particle potential function, which, in the present case, is given by

$$
u_{1}(r)=\left\{\begin{array}{lc}
0 ; & r \leqslant R-d / 2 \\
\infty ; & r>R-d / 2
\end{array} .\right.
$$

From Eqs. (A2) and (A3) we obtain

$$
\Xi(V, T, \mu)=1+z V^{*} ; \quad d / 2<R<d,
$$

where

$$
V^{*} \equiv(4 \pi / 3)(R-d / 2)^{3}
$$

is the volume available to a particle inside the pore, and $z$ is the activity defined by Eq. (4.9). The one-particle distribu- tion function $\rho(\mathbf{r})$ may, in this case, be obtained from

$$
\rho(\mathbf{r})=\frac{z \exp \left[-\beta u_{1}(\mathbf{r})\right]}{\Xi(V, T, \mu)} ; \quad d / 2<R<d .
$$

Combination of Eqs. (A4) and (A6) finally yields

$$
\rho(\mathbf{r})=\rho_{m} g_{p m}(\mathbf{r})=\frac{z \exp \left[-\beta u_{1}(\mathbf{r})\right]}{1+z V^{*}} ; d / 2<R<d .
$$

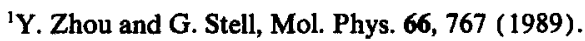

${ }^{2}$ (a) Y. Zhou and G. Stell, Mol. Phys. 66, 791 (1989); (b) 68, 1265 (1989).

${ }^{3}$ D. J. Adams, Mol. Phys. 28, 1241 (1974).

${ }^{4}$ L. S. Ornstein and F. Zernike, Proc. Acad. Sci. (Amsterdam) 17, 793 (1914).

${ }^{5}$ E. D. Glandt, J. Colloid Interface Sci. 77, 512 (1980).

6J. K. Percus and G. J. Yevick, Phys. Rev. 100, 1 (1958).

${ }^{7}$ M. S. Wertheim, Phys. Rev. Lett. 10, 321 (1963),

${ }^{8}$ (a) J. M. J. van Leeuwen, J. Groeneveld, and J. DeBoer, Physica 25, 792 (1959); (b) E. Meeron, J. Math. Phys. 1, 192 (1960).

${ }^{9}$ N. F. Carnahan and K. E. Starling, J. Chem. Phys. 51, 635 (1969).

${ }^{10} \mathrm{~W}$. Feller, An Introduction to Probability Theory and its Applications (Wiley, New York, 1950).

"D. A. McQuarrie, Statistical Mechanics (Harper \& Row, New York, 1976). 